\title{
An AFM study of solid-phase bilayers of unsaturated PC lipids and the lateral distribution of the transmembrane model peptide WALP23 in these bilayers
}

\author{
F. Yarrow
}

Received: 10 January 2011 / Revised: 14 March 2011 / Accepted: 16 March 2011 / Published online: 2 April 2011

(C) The Author(s) 2011. This article is published with open access at Springerlink.com

\begin{abstract}
An altered lipid packing can have a large influence on the properties of the membrane and the lateral distribution of proteins and/or peptides that are associated with the bilayer. Here, it is shown by contact-mode atomic force microscopy that the surface topography of solid-phase bilayers of PC lipids with an unsaturated cis bond in their acyl chains shows surfaces with a large number of line-type packing defects, in contrast to the much smoother surfaces observed for saturated PC lipids. Di- $n$ :1-PC $(n=20,22,24)$ and (16:0,18:1)-PC (POPC) were used. Next, the influence of an altered lipid environment on the lateral distribution of the single $\alpha$-helical model peptide WALP23 was studied by incorporating the peptide in the bilayers of di- $n: 1-\mathrm{PC}$ ( $n=20,22,24)$ and $(16: 0,18: 1)$-PC unsaturated lipids. The presence of WALP23 leads to an increase in the number of packing defects but does not lead to the formation of the striated domains that were previously observed in bilayers of saturated PC lipids and WALP. This is ascribed to the less efficient lateral lipid packing of the unsaturated lipids, while the increase in packing defects is probably an indirect effect of the peptide. Finally, the fact that an altered lipid packing affects the distribution of WALP23 is also confirmed in an additional experiment where the solvent
\end{abstract}

F. Yarrow

Condensed Matter and Interfaces,

Debye Institute for Nanomaterials Science, Science Faculty,

Utrecht University, P.O. Box 80.000,

3508 TA Utrecht, The Netherlands

Present Address:

F. Yarrow $(\bowtie)$

School of Physics, University College Dublin,

Belfield, Dublin 4, Ireland

e-mail: yarrow100@gmail.com
TFE (2,2,2-trifluorethanol) is added to bilayers of di-16:0PC/WALP23. At 3.5 vol\% TFE, the previous striated ordering of the peptide is abolished and replaced by loose lines.

Keywords Protein-lipid interaction - AFM .

Transmembrane model peptide $\cdot$ Striated phase .

Lipid packing $\cdot$ Model membrane

\section{Introduction}

Changes in the properties of the constituting lipid molecules can lead to substantially different properties of the membrane they pack in. For instance, chemical changes in the polar moiety or acyl chains can alter the preferred phase of the bilayer and this can, in turn, affect the mechanical properties (Marsh 1990; Yeagle 2005). Distribution, conformation, and functioning of proteins in the membrane are also affected by changes in the type and phase of lipids (Dunphy and Linder 1998; Shao et al. 1996; Lee 2004; Van den Brink-Van der Laan et al. 2004; McElhaney 1986). These interactions provide a pathway by which biological relevant processes in cells can be steered, such as switching a protein from a nonactive to an active state or compartmentalizing proteins in the correct part of the cell.

In this study, atomic force microscopy (AFM) is used to observe the surface topographies of solid-phase bilayers of PC lipids with an unsaturated cis bond in their acyl chain in the solid phase. The characteristic features in the topographies can be correlated to packing behavior of the lipids in the bilayer and are distinctly different from those of saturated PC lipids in the same phase. The difference is specifically attributed to the perturbing influence of the inflexible cis bond on the overall packing in the bilayer (Cevc 1991). Symmetrically, di-unsaturated di-n:1-PCs (with $n=20,22$, 
or 24 carbon units) and the asymmetric mono-unsaturated POPC [sn1-(16:0)-sn2-(18:1)-PC] were used. These all contain a cis double bond between the 9th and 10th carbon unit from the chain terminus. Most lipids in biological membranes contain one or more $c i s$ unsaturated bonds (Marsh 1990). This study constitutes the first AFM study of these lipids in the solid phase and might help to complement the sparse information available on these systems. While the solid phase used here is not biologically the most relevant, it still serves to demonstrate general issues of lipid packing. Effects of packing may be expected to be more pronounced in the rigidly and orderly packed solid phase than in the more loosely organized fluid phase. Moreover, it is necessary to use this phase to allow comparison with the peptide-containing bilayers (see below) from the literature.

The effect of these lipids on the lateral distribution of the model peptide WALP23 is also investigated here, again by AFM. This peptide has been used successfully in the past in biophysical studies concerning direct lipid-peptide interactions and hydrophobic mismatch (De Planque et al. 1998; De Planque and Killian 2003; Killian et al. 1996; Rinia et al. 2000). The peptide was modelled to mimic one of the $\alpha$-helical, transmembrane units that are often found in membrane-spanning proteins and can therefore provide insight into the factors regulating the organization of protein subunits in the membrane. Interestingly, insertion of WALP into gel-phase bilayers of saturated PC lipids leads to self-assembled, regular patterns of alternating rows of WALP and rows of lipids with a modified conformation (De Kruijff et al. 2006; Sparr et al. 2005). The driving forces for the formation of this so-called striated phase are believed to involve direct lipid-peptide interactions and packing requirements of the lipids in the bilayer. The importance of packing requirements was underlined by the observed disaggregation of WALP in saturated PC bilayers from lines to smaller units upon going from the gel to the disordered liquid-crystalline phase (Sparr et al. 2005; Scarpelli et al. 2009). It may equally be expected that other changes in the lipid matrix, as introduced through the presence of the cis bond, will also lead to changes in the formation of the lateral distribution of the WALP23. However, up till now, saturated PC lipids have exclusively been used in the AFM studies (Rinia et al. 2000; De Kruijff et al. 2006). The results of this study could serve to enhance our understanding of the exact contribution of all involved driving forces determining lateral distribution of proteins.

Finally, a different approach is taken to underline the effect of lipid packing on the distribution of WALP23, i.e., by adding the solvent TFE (2,2,2-trifluorethanol) to mixed bilayers of WALP23 and the saturated PC lipid DPPC (di-16:0-PC). The presence of TFE alters properties of the bilayer as the TFE molecules partition selectively into the PC polar region (Van den Brink-Van der Laan et al. 2004; Özdirekcan et al. 2008). The altered distribution of WALP23 at sufficiently high TFE concentrations serves as additional proof of the importance of the packing of the lipids in the bilayer.

\section{Materials and methods}

\section{Materials}

All lipids were purchased from Avanti Polar Lipids, Alabaster, AL, USA at $>99.0 \%$ purity and were used as received. Chain-unsaturated DeiPC (1,2-dieicosenoyl$s n$-glycero-3-phosphocholine $=$ di-20:1c11-PC, with $n=20$ carbon atoms in the chain and $m=1$ unsaturated bond in the cis conformation at position C11), DerPC (1,2-dierucoyl$s n$-glycero-3-phosphocholine $=$ di-22:1c13-PC), DNPC $(1,2-$ dinervonoyl-sn-glycero-3-phosphocholine $=$ di-24:1c15-PC), the mono-unsaturated POPC (1-palmitoyl-2-oleoyl-sn-glycero-3-phosphocholine $=16: 0,18: 1 c 9-\mathrm{PC})$, and the chain-saturated DPPC (1,2-dipalmitoyl-sn-glycero-3-phosphocholine = di-16:0-PC) were used. The solid phase of these long-chained, unsaturated PC lipids is more accessible than that of their shorter analogues, whose main transition temperatures, $T_{\mathrm{M}}$, typically lie below $-10^{\circ} \mathrm{C}$ (Marsh 1990). More specifically, $T_{\mathrm{M}}$ is $-4.3^{\circ} \mathrm{C}$ for di-20:1-PC, $13.2^{\circ} \mathrm{C}$ for di-22: 1-PC, $26.7^{\circ} \mathrm{C}$ for di-24:1-PC, and $-0.8^{\circ} \mathrm{C}$ for $(16: 0,18: 1)-\mathrm{PC}$ (Marsh 1990).

WALP23 was a gift from Prof. Dr. J. A. Killian (Dept. Biochemistry of Membranes, Utrecht University). The amino acid sequence of WALP23 is acetyl- $\mathrm{GW}_{2} \mathrm{~L}(\mathrm{AL})_{8}$ $\mathrm{W}_{2} \mathrm{~A}-\mathrm{NH}_{2}$, where $\mathrm{A}=$ alanine, $\mathrm{W}=$ tryptophan, $\mathrm{L}=$ leucine, $\mathrm{G}=$ glycine amino acids. The alanine and leucine groups form a highly hydrophobic $\alpha$-helix with a length $D_{\mathrm{WALP}}=2.6 \mathrm{~nm}$. WALP adopts a transmembrane orientation in the bilayer, anchored by the tryptophan groups (De Planque and Killian 2003; Contera et al. 2005). WALP at $2 \mathrm{~mol} \%$ was used in all experiments, unless stated otherwise.

Preparation of the bilayers

Supported bilayers were prepared according to the vesicle fusion protocol (Brian and McConnell 1984). Lipids were dissolved in a solution of $1: 3 \mathrm{v} / \mathrm{v}$ chloroform/methanol (analytical grade), and WALP23 was dissolved in TFE (2,2,2-trifluorethanol, Sigma-Aldrich, 99.5+\% NMR grade). The vesicle dispersions and supported bilayers were then prepared essentially as described by Rinia et al. (2000), but the $10 \mathrm{~h}$ waiting period was omitted. Dispersions were stored at $5^{\circ} \mathrm{C}$ if not used immediately but were not kept for more than 3 days. All samples were made and measured at least twice to ensure reproducibility. The aqueous 
hydrating and measuring medium in most samples was $20 \mathrm{mM} \mathrm{NaCl}$ (Merck Darmstadt, Germany, p.a. grade; in MilliQ-water of $>18 \mathrm{M} \Omega / \mathrm{cm}$ ), following Rinia et al. (2000). Salinity was increased, however, to $2 \mathrm{M}$ for di20:1-PC and (16:0,18:1)-PC to prevent the formation of ice at the low measuring temperatures used. $\mathrm{NaCl}$ at $2 \mathrm{M}$ depresses the freezing point of water by $8^{\circ} \mathrm{C}$ (Lide 2001). It was checked that $2 \mathrm{M} \mathrm{NaCl}$ did not alter the morphology of the striated domains in di-16:0-PC/2 mol\% WALP23 bilayers and gave a comparable repeat distance between the stripes (i.e., $7.5 \pm 0.3 \mathrm{~nm}$ at $2 \mathrm{M}$ and $7.7 \pm 0.6 \mathrm{~nm}$ at $20 \mathrm{mM}$ ). $\mathrm{NaCl}$ should indeed leave the membrane structure and bending rigidity unmodified (Petrache et al. 2006), although altered AFM probe-sample interactions led to an apparent increase in the measured bilayer thickness of $\sim 15 \%$ for di-16:0-PC at $2 \mathrm{M} \mathrm{NaCl}$, in agreement with Müller and Engel (1997).

The TFE-containing bilayers were made by adding small amounts (1-5 vol\%) of the solvent TFE to di-16:0-PC and di-16:0-PC/2 mol\% WALP23 vesicle suspensions prior to applying them to the mica substrate. Imaging was done in $20 \mathrm{mM} \mathrm{NaCl}$ with the same $1-5$ vol\% TFE content.

\section{AFM measurements}

AFM was performed in contact mode with a commercial PicoScan Microscope (Molecular Imaging, Phoenix, AZ, USA, model 305-0002 157) and the S-1286 scanner. Measurements were performed in the solid phase at $13^{\circ} \mathrm{C}$ for di24:1-PC, $4^{\circ} \mathrm{C}$ for di-22:1-PC, and $-6^{\circ} \mathrm{C}$ for di-20:1-PC and (16:0,18:1)-PC, by mounting the sample on a Peltier I or II $3 \times$ sample stage (Molecular Imaging) that was connected to a LakeShore 330 Temperature Controller. The samples were cooled from room temperature to the desired measuring temperature at a rate of $1^{\circ} \mathrm{C} / \mathrm{min}$, unless stated otherwise. The temperature on top of the mica disk immersed in $\mathrm{NaCl}$ solution was calibrated with a Pt-100 sensor (Sensycon, Alzenau, Germany) with a flat metal disk ( $\varnothing 4.8 \mathrm{~mm}$ ) as probe. Calibrated values are used throughout.

Pyramid-shaped $\mathrm{Si}_{3} \mathrm{~N}_{4}$ oxide-sharpened tips (NP-S, NanoProbe, Digital Instruments, Santa Barbara, CA, USA) mounted on a triangular cantilever with a nominal spring constant of $0.06 \mathrm{~N} / \mathrm{m}$ and a typical radius of 5-20 nm were used. A silicon calibration grid with ridges of a step height of $24 \mathrm{~nm}$ and a width of $3 \mu \mathrm{m}$ (TGZ01 UltraSharp grating set, NT-MDT, Moscow, Russia) was used for calibration of the AFM scanner. Scanning was done at the lowest possible force that allowed stable imaging, typically around $0.4 \mathrm{nN}$. This was maintained by decreasing the force setpoint manually during the scan, as thermal drift can cause deviations in the actual force. A scan rate of 3 lines/s was used. All presented images are in the height, topographic mode and are background-corrected ("flattened") with the use of the
PicoScan 5.2 software (Molecular Imaging, Phoenix, AZ, USA). The relative height of the features in the images is represented by a gray scale, where a lighter color indicates a greater height. The total height scale in the presented images corresponds to a difference in height of $\sim 1 \mathrm{~nm}$, unless stated otherwise.

The thickness of the bilayer $d_{\text {bil }}$ is defined as the difference in height between the top of the bilayer and the mica substrate and can be measured through holes piercing the layer. Averages were taken of at least 50 separate measurements.

\section{Results}

Bilayers of unsaturated lipids

Supported, hydrated bilayers of symmetrical, di-unsaturated di- $n$ :1-PCs with $n=20,22$, and 24 showed comparable topological features in the low-temperature solid state (see Fig. 1a for $n=22$ ). Terraces were observed whose size was estimated to be $50-275 \mathrm{~nm}$ for $n=22$. The edges of these domains exhibit a long-range directional order. Separate regions can be observed where the overall orientation of the domains is rotated at angles close to $60^{\circ}$ or $120^{\circ}$ with respect to each other. In Fig. 1a, three separate regions are present: at the top left, in the middle, and at the bottom right. Each separate region probably originates from a different solid-phase nucleus that was formed upon crystallization from the fluid phase.

Lines with a depth of $0.5-1 \mathrm{~nm}$ marked the borders of the crystalline domains. The width of these lines was $6 \mathrm{~nm}$ at low scanning forces of $\leq 0.5 \mathrm{nN}$. These lines will be considered line-type packing defects. The initial number of defects that is observed when the first solid phase appears (in the coexistence region in Fig. 1b) does not alter upon further cooling or within the time span of the measurements (approximately a few hours). A useful and definable quantity to compare domain sizes between the different lipid bilayers is the density of these packing defects, $\rho_{\text {line }}$ (三 cumulative length of the lines per area). These are given in Table 1. $\rho_{\text {line }}$ clearly decreases with $n$. It is assumed that $\rho_{\text {line }}$ correlates with the stability of the packing (see section "Bilayers of unsaturated lipids"). The $\rho_{\text {line }}$ of the di-24:1PC bilayer was independent (within the error) of the cooling rate $\left(0.2-40^{\circ} \mathrm{C} / \mathrm{min}\right)$ at which the bilayer was cooled from the fluid to the low-temperature phase and did not alter after reaching the solid state, even when the bilayer was cooled further. The morphology of these bilayers is distinctly different from gel-state bilayers of saturated PC lipids, such as di-16:0-PC, where smooth layers with a small number of meandering line-type packing defects are observed, with a typical $\rho_{\text {line }}$ of $1 \mu \mathrm{m} / \mu \mathrm{m}^{2}$ (Yarrow and Kuipers 2011; Rinia et al. 2000). 


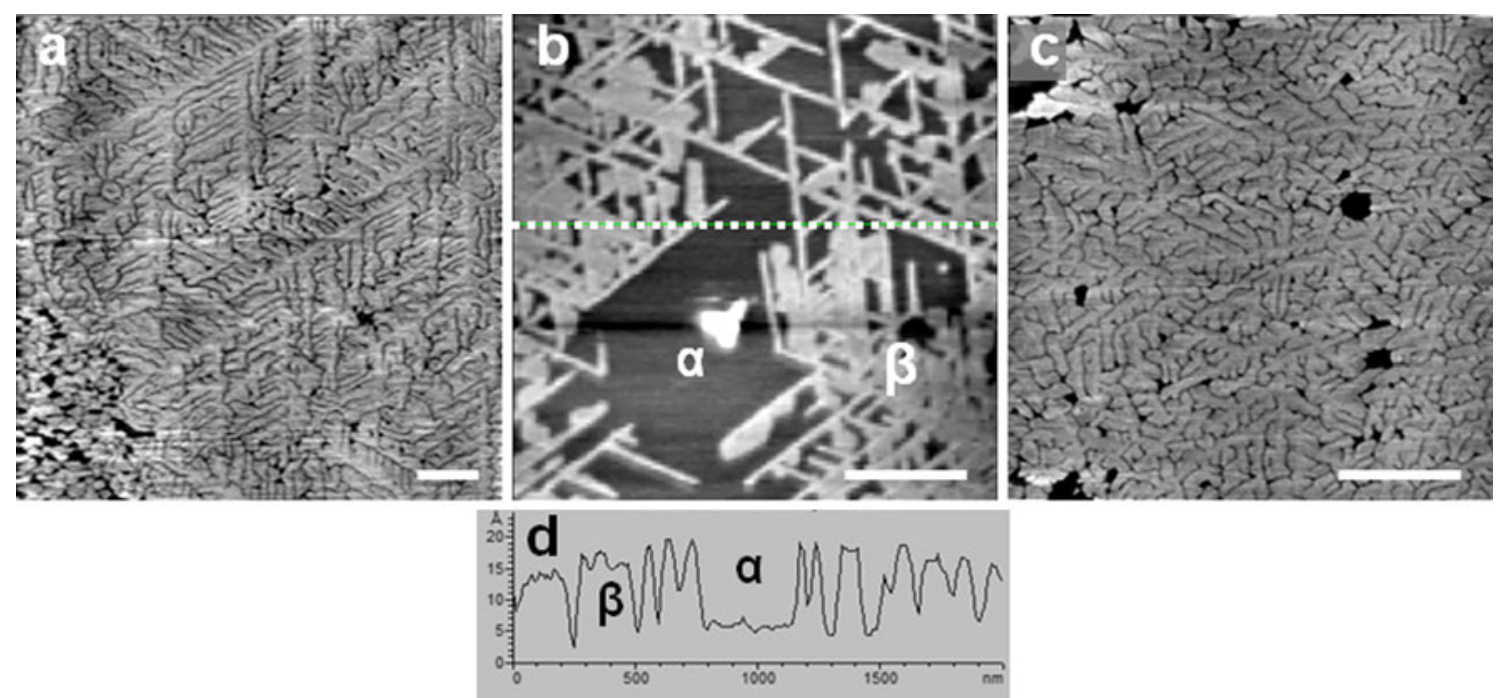

Fig. 1a-d AFM topographs of a di-22:1-PC $(n=22)$ bilayer. a In the solid state at $4{ }^{\circ} \mathrm{C}$. The holes at the bottom left part of the scan are due to damage from a previous scan at a higher scanning force. $\mathbf{b}$ In the coexistence region at $7^{\circ} \mathrm{C}$, which shows a fluid phase, marked by $\alpha$, and

Table 1 Line densities, $\rho_{\text {line }}\left(\mu \mathrm{m} / \mu \mathrm{m}^{2}\right)$, for the different unsaturated lipids with and without WALP

\begin{tabular}{llccl}
\hline $\begin{array}{l}\text { Initial } \\
\text { WALP2 } \\
(\mathrm{mol} \%)\end{array}$ & di-20:1-PC & di-22:1-PC & di-24:1-PC & $(16: 0,18: 1)-P C$ \\
\hline 0 & 10 & 6 & 4 & 27 \\
0.5 & - & - & 5 & - \\
1 & - & - & 10 & - \\
2 & $-^{\mathrm{a}}$ & 14 & 8 & 90 \\
4 & - & - & 10 & - \\
\hline
\end{tabular}

The error in $\rho_{\text {line }}$ is $\sim 2 \mu \mathrm{m} / \mu \mathrm{m}^{2}$ (based on five separate measurements of different areas of the sample)

${ }^{a}$ Poor quality of the scans hindered a reliable determination

The bilayer thickness $d_{\text {bil }}$ of the bilayers with $n=20,22$, and 24 was respectively $6.3 \pm 0.4,6.3 \pm 0.3$, and $6.6 \pm 0.4 \mathrm{~nm}$. These numbers fall short of the predicted increase in acyl length of $0.45 \mathrm{~nm}$ per 2 methyl additions (Dumas et al. 1999), which could be caused by differences in tilt angle, ionic strength, and small differences in applied force of the AFM probe. Overall, the values of $d_{\mathrm{bil}}$ are close to those of saturated lipids and should similarly indicate the presence of bilayers in the solid phase with a layer of interstitial water between the bilayer and mica substrate (Rinia et al. 2000; Marsh 1990; Shao et al. 1996).

Only one transition was observed upon cooling the sample from a higher temperature. Coexisting areas of the fluid and solid are shown in Fig. $1 \mathrm{~b}$ for $n=22$ at $7^{\circ} \mathrm{C}$. The former is recognizable by its reduced height of $\sim 1 \mathrm{~nm}$ compared to the solid phase (Fig. 1d). This reflects the increased disorder of the acyl chains in the fluid phase a solid phase, marked by $\beta$. The corresponding height profile in $\mathbf{d}$ is recorded at the position of the white dotted line. $\mathbf{c}$ Mixed di-22:1-PC/ $2 \mathrm{~mol} \%$ WALP23 bilayer in the solid state at $4{ }^{\circ} \mathrm{C}$. Scale bars are $500 \mathrm{~nm}$ in each image

(Williams et al. 1996). Bilayers of (16:0,18:1)-PC (POPC) showed distinct patterns with a large number of interconnected dark lines with a width $\sim 9-15 \mathrm{~nm}$ and depth $\sim 0.3 \mathrm{~nm}$ (Fig. 2a). The solid domains were smaller $(\sim 30-130 \mathrm{~nm})$ than those of the di- $n: 1-P C$ bilayers, and the bordering lines were broader and showed less preferential orientation. $d_{\text {bil }}$ was $6.1 \pm 0.4 \mathrm{~nm}$.

Mixed bilayers of unsaturated lipids with WALP

Inclusion of $2 \mathrm{~mol} \%$ WALP23 in bilayers of the unsaturated lipid bilayers gave a topology as shown in Figs. 1c and $2 \mathrm{~b}$. This is in contrast with the known behavior of WALP23 in bilayers of saturated PCs, where the presence of WALP23 leads to microdomains with a striated pattern as shown in Fig. 3a (Rinia et al. 2000).

Evidently, no such striated ordering is observed for the unsaturated PCs. In fact, the only discernible change was a decrease in the size of the terraces, which resulted in a (more than) doubling of $\rho_{\text {line }}$ (Figs. 1c, 2b; Table 1). Estimated domain sizes are $35-160 \mathrm{~nm}$ for the $n=22 / 2 \mathrm{~mol} \%$ WALP23 system and 20-60 nm for the (16:0,18:1)-PC/ 2 mol\% WALP23 system. Table 1 shows that the doubling of $\rho_{\text {line }}$ was already achieved at $1 \mathrm{~mol} \%$ WALP23 for $n=24$ and further increases in the concentration of WALP did not lead to additional changes.

TFE-containing bilayers

An additional test was performed to get insight into the effects of the bilayer properties by adding TFE to di-16:0PC and di-16:0-PC/2 mol\% WALP23 bilayers. The bilayer 
Fig. 2 Scan of a (16:0,18:1)-PC (POPC) bilayer a without and b with 2 mol\% WALP23. The apparent absence of structure in the middle of $\mathbf{b}$ was an artefact due to a temporary high scanning force around $1 \mathrm{nN}$. Scale bars are both $250 \mathrm{~nm}$
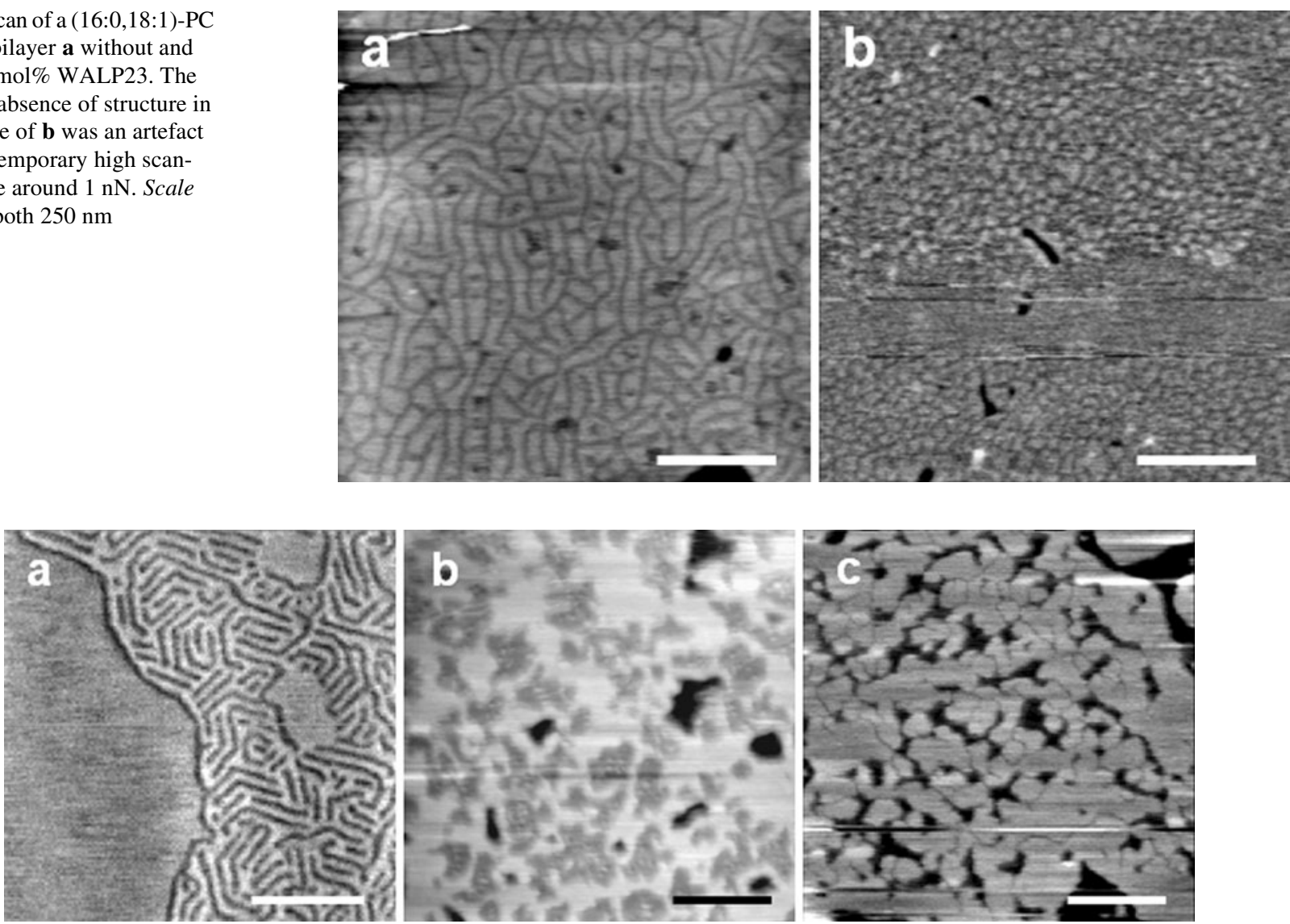

Fig. 3 AFM micrograph of a a di-16:0-PC (DPPC) bilayer with 2 mol\% WALP23 (scale bar $100 \mathrm{~nm}$ ), b di-16:0-PC bilayer with 5 vol\% TFE (scale bar $250 \mathrm{~nm}$, total height scale $5 \mathrm{~nm}$ ), and c di-16:0-PC bilayer with $2 \mathrm{~mol} \%$ WALP23 and 3.5 vol\% TFE (scale bar $250 \mathrm{~nm}$ )

thickness $d_{\text {bil }}$ was 5-5.5 nm for all TFE concentrations from 0 to $3.5 \mathrm{vol} \%$, indicating the continued presence of a single, gel-state bilayer without large conformational changes. For 4 and 5 vol\%, however, domains of a second phase appeared with a reduced height of $\sim 0.7 \mathrm{~nm}$ (Fig. $3 \mathrm{~b}$ ).

Between 0 and $3 \mathrm{~mol} \%$ TFE, striated areas were observed in the mixed WALP-containing di-16:0-PC/TFE bilayers. The properties of the striated domains were unaffected with a repeat distance of 7.5-8 $\mathrm{nm}$ and a domain height of $0.2-0.3 \mathrm{~nm}$. These values are the same as those observed in the absence of TFE (Rinia et al. 2000). At 3.5 vol\%, the striated pattern vanished and was replaced by assemblies of dark lines, as shown in Fig. 3c.

\section{Discussion}

Bilayers of unsaturated lipids

The distinctive features of hydrated, supported bilayers of di-n:1-PC ( $n=20,22$, and 24) and (16:0,18:1)-PC lipids are substantially different from those of saturated PC lipids. To the best of our knowledge, this is the first AFM study on the solid state of these kinds of lipids in a supported bilayer and can complement the sparse information that is available on these systems. Support for the presented data can be found, however, in a Brewster angle microscopy study on condensed Langmuir-Blodgett monolayers of unsaturated erucic (C22:1c13) and nervonic (C24:1c15) fatty acids (Vollhardt 2007), which showed a comparable pattern.

It is clear that the cis bond gives rise to the observed changes in topography compared to saturated PC bilayers. The unsaturated bond has a perturbing influence on the much more rigidly organized solid phase. In hydrated bilayers of di- $n: 1-\mathrm{PCs}$, the packing of the acyl chains is severely hindered by the double bond, and a loosely packed solid phase is formed where the acyl chains are packed with their long axes tilted with respect to the bilayer normal ( $\mathrm{Li}$ et al. 1994; Williams et al. 1996; Macdonald et al. 1985; Lewis et al. 1988). No well-ordered hexagonal packing is formed (Williams et al. 1996). The effect of the kink at the cis bond is minimized to some extent as molecular mechanics calculations have indicated that the surrounding bonds will adapt so that a "crankshaft-like" motif is formed ( $\mathrm{Li}$ et al. 1994; Macdonald et al. 1985). The cis bond causes a decrease in orientational order or increase in motional freedom, 
depending on its exact position within the acyl chain (Macdonald et al. 1985). The area per lipid is larger (Demel et al. 1972), and the efficiency of the packing is less-leading to a lower $T_{\mathrm{M}}$-than for saturated PC lipids, which are packed tightly in the solid gel $\mathrm{L}_{\beta}^{\prime}$ phase with the acyl chains completely parallel to each other and tilted with respect to the bilayer normal (Cevc 1991).

The observed surface topologies of the di- $n$ :1-PC bilayers consist of a relatively large number of solid domains that are most likely bordered by packing defects (Litman et al. 1991). The number of packing defects as expressed by $\rho_{\text {line }}$ is higher than for di-16:0-PC (DPPC) at approximately the same cooling rate $\left[4-10 \mu \mathrm{m} / \mu \mathrm{m}^{2}\right.$ for the di- $n: 1-\mathrm{PCs}$ and $27 \mu \mathrm{m} / \mu \mathrm{m}^{2}$ for $(16: 0,18: 1)$-PC versus $1 \mu \mathrm{m} / \mu \mathrm{m}^{2}$ for di-16:0-PC]. Defects generally arise in order to relieve packing frustrations. Packing strain is expected to be higher for the unsaturated chains with their inflexible bend that hinders positioning of the molecules according to the longrange translational order of the crystalline phase. Moreover, dendrite-like patterns, such as those observed here for di- $n: 1-\mathrm{PC}$, usually result from limiting transport dynamics during solidification (Blanchette et al. 2008; Langer 1980). Diffusion in the fluid phase (Metso et al. 2004) and proper alignment of a kinked lipid with the growing solid phase are slower than for saturated PC lipids, leading to these dendritical patterns. This seems to be supported by the independency of $\rho_{\text {line }}$ of di-24:1-PC with the rate of cooling, indicating that nucleation is not rate-determining during solidification; again unlike bilayers of the saturated di-16:0-PC where nucleation rate governs the kinetics (Yarrow and Kuipers 2011).

The AFM topographs of the solid phase of the unsaturated PC lipids do not provide information on the precise nature of the solid phase. Only one solid phase was found upon cooling of the $\mathrm{L}_{\alpha}$ phase.

Previous studies (Lewis et al. 1988; Williams et al. 1996) showed that the initially formed gel phase transforms relatively quickly into a loosely packed subgel-like phase with very restricted phosphate-group motions. This is distinctly different from saturated PC lipids such as di-16:0$\mathrm{PC}$, where the subgel phase forms only after prolonged annealing at low temperatures (Füldner 1981).

Probably, the solid phase observed in this AFM study is the subgel-like phase as the measuring temperatures here fall in the region where DSC indicates that the subgel-like phase is stable, i.e., below the lowest exotherm (Lewis et al. 1988). The transition from gel to this subgel phase might fall within the few minutes it takes to complete the AFM scan or, alternatively, might not be discernible as the accompanying changes in height are very small. For di-16:0-PC bilayers, the lamellar repeat periodicity only changes from $6.3 \mathrm{~nm}$ in the gel to $5.9 \mathrm{~nm}$ in the subgel phase (Füldner 1981).
Surfaces of bilayers of the mono-unsaturated (16:0,18:1)-PC (POPC) show a different pattern and have a larger $\rho_{\text {line }}$ than those of the di- $n: 1-\mathrm{PCs}$. The asymmetry between the oleoyl and palmitoyl chain for POPC makes parallel alignment of the chains more difficult than for two symmetrical, unsaturated chains (Yun et al. 2003). Computer simulations show that at low temperatures the saturated $s n-1$ 16:0 hydrocarbon chain is fully extended and packs with a tilt angle with respect to the bilayer normal in a gel $\mathrm{L}_{\beta}^{\prime}$ phase, whereas the unsaturated $s n-2$ 18:1 chain can pack only partially parallel to the $s n-1$ chain (Lee et al. 1995; Leekumjorn and Sum 2007). The larger strain on the packing is relieved through a higher number of packing defects. Of course, different growth/nucleation dynamics or a different origin of the lines could also affect domain size. A possibility is that the two opposing leaflets are (partially) interdigitated (Leekumjorn and Sum 2007), as can also occur for other asymmetric mixed-chain lipids (Hui et al. 1984).

\section{Mixed bilayers of unsaturated lipids with WALP}

The only morphological change upon incorporation of WALP23 in bilayers of the unsaturated lipids is a refinement of the pattern (and hence $\rho_{\text {line }}$ ) that was observed without the peptide.

The value of $\rho_{\text {line }}$ becomes independent of the added amount of WALP23 above $1 \mathrm{~mol} \%$ (for $n=24$ ). Previous studies have indicated limited incorporation of WALP in bilayers of saturated, long-chained PC lipids (De Planque and Killian 2003). This might also be the case here, where the amount of WALP23 is probably limited around $1 \mathrm{~mol} \%$. The excluded WALP23 could be present in patches of debris that were occasionally observed on the bilayer surface (not shown).

The incorporated peptide molecules could in principle be present in the packing defects and/or dissolved in the bilayer. WALP23 is known to accumulate in packing defects of di-16:0-PC bilayers (Rinia et al. 2000) at low concentrations. This might occur here to some extent, although the number of packing defects is only sufficient to accommodate maximally $0.2-0.3 \mathrm{~mol} \%$ WALP $23,{ }^{1}$ where it can be reasonably assumed that the intrinsic properties of WALP23 and/or specific lipid-WALP interactions only allow for the formation of single lines, as was shown to occur in saturated PC bilayers (De Planque and Killian

\footnotetext{
$\overline{{ }^{1} \mathrm{~A} \rho_{\text {line }} \text { of } 8-10} \mu \mathrm{m} / \mu \mathrm{m}^{2}$ for $n=24$ can incorporate $8-10 \times 10^{3}$ WALP molecules per $\mu \mathrm{m}^{2}$ surface area when using a diameter of $1 \mathrm{~nm}$ for WALP (Sparr et al. 2005) and assuming single rows, and $1 \mu \mathrm{m}^{2}$ is covered by $1.6 \times 10^{6}$ lipid molecules [area/lipid $=0.6 \mathrm{~nm}^{2}($ Marsh 1990)] The actual number of lipids is double owing to the bilayer nature. This leads to $0.2-0.3 \mathrm{~mol} \%$ WALP23.
} 
2003; Sparr et al. 2005; Contera et al. 2005). At least part of the $\sim 1 \mathrm{~mol} \%$ WALP23 must therefore be located elsewhere and is possibly distributed randomly throughout the lipid phase in the form of single molecules or di- or trimers that are too small to be detected by AFM.

A different situation exists for (16:0,18:1)-PC/WALP23 bilayers, where $\rho_{\text {line }}$ is theoretically large enough to accommodate 2.7 mol\% WALP. All WALP could then be located in the observed lines, although this does not explain why the pattern of the lines is altered in the presence of WALP. Possibly (partly dissolved) WALP23 acts indirectly on all studied unsaturated lipid systems, for instance by altering mechanical properties of the membrane (Vitovič et al. 2004; Siegel et al. 2006) or by altering the kinetics of nucleation and growth (Rauls et al. 2000).

It is instructive to compare these results with those of WALP23 in gel-phase di-16:0-PC bilayers, where peptide molecules that are not accommodated in the packing defects are excluded to separate areas of a striated phase (De Kruijff et al. 2006; Fig. 3a). In the striated phase, alternating single rows of WALP alternated with rows of four to five lipids whose conformation is affected by the peptide. Low solubility opposed by a mixing tendency caused by strong lipid-peptide interactions is believed to be the driving force for these striped patterns (De Kruijff et al. 2006).

The relatively loose packing of the unsaturated lipid acyl chains in the bilayer probably facilitates solubility of the peptide as compared to saturated PCs. This looser packing is confirmed by the larger area per lipid $\left[\sim 45-50 \AA^{2}\right.$ for saturated di-16:0-PC and $\sim 55-60 \AA^{2}$ for $(16: 0,18: 1)$-PC (Yun et al. 2003)] and larger average chain ordering $S$ of $\sim 0.75$, which is closer to that of a saturated palmitoyl chain in the fluid phase $(S=0.65)$ than in the gel phase $(S=0.9)$ (Binder and Gawrisch 2001). In fact, enhanced solubility and reduced lateral ordering of WALP were also found in fluid di-16:0-PC bilayers (Sparr et al. 2005). A lesser extent of aggregation of WALP was also found by EPR in solid bilayers of di-18:1-PC than in bilayers of di-16:0-P, while no aggregation was found in the fluid phase of both these lipids (Scarpelli et al. 2009). This is related to the higher extent of perturbation the peptide molecules exert on a more rigidly organized bilayer than on a more disordered one. Hence, the energetic gain obtained by localization of the peptide to the striated phase is highest in gel-phase bilayers of saturated PC lipids.

\section{TFE-containing bilayers}

The effect of lipid packing on the lateral ordering of WALP is underlined by the TFE experiments.

First, the topology of pure di-16:0-PC bilayers is shown to be altered by TFE as a second phase with a reduced thickness is formed at 4 and 5 vol\% TFE. This is probably an interdigitated phase as is also formed in the presence of other small alcohols (Mou et al. 1994). TFE molecules partition selectively in the polar head group region and induce an increase in the head group area. This leads to a disordering of the acyl chains (Van den Brink-Van der Laan et al. 2004; Özdirekcan et al. 2008), which can apparently interdigitate at sufficiently high concentrations, to the formation of an interdigitated phase.

In the di-16:0-PC/WALP23 systems, concentrations of TFE at $\leq 3$ vol\% do not affect the striated patterns in the WALP23-containing bilayers. However, sufficiently large organizational modifications in the bilayer take place at 3.5 vol\% TFE where the striated phase is replaced by loose lines. The width of the lines does suggest that these are single rows of WALP23 (see above). The most plausible explanation for the reduced lateral ordering could be the TFE-induced disordering of the lipid's acyl chains (Van den Brink-Van der Laan et al. 2004), where the ordering is still high enough to allow complete disaggregation of WALP, but too low to form ordered striated domains. TFE was also shown to lead to the dissociation of the subunits of the tetrameric membrane protein KcsA (Van den BrinkVan der Laan et al. 2004).

A second possibility is that TFE interferes with the interactions between the anchoring tryptophan groups of the peptide and the lipid bilayer as shown in a ${ }^{2} \mathrm{H}$ NMR study of WALP in a fluid di-14:0-PC bilayer (Özdirekcan et al. 2008). It is not certain how substantial this effect is in the gel phase, however. A reduction in the tryptophan-lipid interaction energy could have consequences for the lipids bordering the peptide and, through this, the striped ordering. On the other hand, replacement of the tryptophan groups with other uncharged, aromatic amino acid moieties did not change the ability of WALP to form a striated phase (Rinia et al. 2002), while lipid packing has a large effect (Sparr et al. 2005; Yarrow and Kuipers 2011) so it could be speculated that changes in the peptide-lipid interactions are of lesser importance than changes in lipid packing for the formation of a striated phase. Additionally, single lines of peptide are still formed at $3.5 \mathrm{vol} \%$ TFE, while weakening of the lipid-peptide interactions is expected to lead to larger aggregates of WALP23.

\section{Concluding remarks}

In this study, it was shown that packing properties of the constituting lipids can substantially affect the morphology and growth kinetics in supported bilayers. The packing, and especially the "tightness" of the packing, can be altered through chemical modifications of the lipids themselves (i.e., introduction of a cis bond in the acyl chain) or through the use of small molecules that incorporate in the bilayer 
and affect packing. Lipid packing is also shown to be a determinant of the lateral distribution of peptides in membranes. Conversely, the presence of peptide clearly alters the morphology of the (unsaturated) bilayers in the case of unsaturated lipids.

Although the solid phase used in this study may not be the most interesting from a biological perspective, from a physical perspective, studies of this phase allow unique insight into the mechanisms by which the overall packing properties of biological membranes can be altered as well as the lateral distribution of lipids. The observed effects are limited to these small model peptides. WALP is a model of a single $\alpha$-helical segment, of which virtually all membrane-spanning proteins contain a number. The results concerning the lipid-related aggregation of WALP can therefore be extended to changes in the relative position of these subunits, which can determine whether a protein is in an active, functioning or inactive mode. This is highly relevant from a biologically point of view.

Acknowledgments This research was funded by the Netherlands Organization for Scientific Research (NWO). Thanks to Prof. Dr. J.A. Killian for providing the WALP and Pa-WALP and to Prof. Dr. B. de Kruijff for helpful discussions and ideas. B.W.M. Kuipers M.Sc. is thanked for his useful comments on the manuscript.

Open Access This article is distributed under the terms of the Creative Commons Attribution Noncommercial License which permits any noncommercial use, distribution, and reproduction in any medium, provided the original author(s) and source are credited.

\section{References}

Binder H, Gawrisch K (2001) Effect of unsaturated lipid chains on dimensions, molecular order and hydration of membranes. J Phys Chem B 105:12378-12390

Blanchette CD, Orme CA, Ratto TV, Longo ML (2008) Quantifying growth of symmetric and asymmetric lipid bilayer domains. Langmuir 24:1219-1224

Brian AA, McConnell HM (1984) Allogeneic stimulation of cytotoxic T cells by supported planar membranes. Proc Natl Acad Sci USA 81:6159-6163

Cevc G (1991) How membrane chain-melting phase-transition temperature is affected by the lipid chain asymmetry and degree of unsaturation: an effective chain-length model. Biochemistry 30:7186-7193

Contera SA, Lemaître V, De Planque MRR, Watts A, Ryan JF (2005) Unfolding and extraction of a transmembrane $\alpha$-helical peptide: dynamic force spectroscopy and molecular dynamics simulations. Biophys J 89:3129-3140

De Kruijff B, Killian JA, Ganchev DN, Rinia HA, Sparr E (2006) Striated domains: self-organizing assemblies of transmembrane $\alpha$-helical peptides and lipids in bilayers. Biol Chem 387:235-241

De Planque MRR, Killian JA (2003) Protein-lipid interactions studied with designed transmembrane peptides: role of hydrophobic matching and interfacial anchoring (review). Mol Membr Biol 20:271-284

De Planque MRR, Greathouse DV, Koeppe RE II, Schäfer H, Marsh D, Killian JA (1998) Influence of lipid/peptide hydrophobic mismatch on the thickness of diacylphosphatidylcholine bilayers. A ${ }^{2} \mathrm{H}$ NMR and ESR study using designed transmembrane $\alpha$-helical peptides and gramicidin A. Biochemistry 37:9333-9345

Demel RA, Geurts van Kessel WSM, Van Deenen LLM (1972) The properties of polyunsaturated lecithins in monolayers and liposomes and the interactions of these lecithins with cholesterol. Biochim Biophys Acta 266:26-40

Dumas F, Lebrun M-C, Tocanne J-F (1999) Is the protein/lipid hydrophobic matching principle relevant to membrane organization and functions? FEBS Lett 458:271-277

Dunphy JT, Linder ME (1998) Signalling functions of protein palmitoylation. Biochim Biophys Acta 1436:245-261

Füldner HH (1981) Characterization of a third phase transition in multilamellar dipalmitoyllecithin liposomes. Biochemistry 20:57075710

Hui SW, Mason JT, Huang C (1984) Acyl chain interdigitation in saturated mixed-chain phosphatidylcholine bilayer dispersions. Biochemistry 23:5570-5577

Killian JA, Salemink I, De Planque MRR, Lindblom G, Koeppe RE II, Greathouse DV (1996) Induction of nonbilayer structures in diacylphosphatidylcholine model membranes by transmembrane $\alpha$-helical peptides: importance of hydrophobic mismatch and proposed role of tryptophans. Biochemistry 35:1037-1045

Langer JS (1980) Instabilities and pattern formation in crystal growth. Rev Mod Phys 52:1-28

Lee AG (2004) How lipids affect the activities of integral membrane proteins. Biochim Biophys Acta 1666:62-87

Lee B, Mabry SA, Jonas A, Jonas J (1995) High-pressure proton NMR study of lateral self-diffusion of phosphatidylcholines in sonicated unilamellar vesicles. Chem Phys Lipids 78:103-117

Leekumjorn S, Sum AK (2007) Molecular characterization of gel and liquid-crystalline structures of fully hydrated POPC and POPE bilayers. J Phys Chem B 111:6026-6033

Lewis RNAH, Sykes BD, McElhaney RN (1988) Thermotropic phase behavior of model membranes composed of phosphatidylcholines containing cis-monounsaturated acyl chain homologs of oleic acid: differential scanning calorimetric and phosphorus-31 NMR spectroscopic studies. Biochemistry 27:880-887

Li S, Lin H, Wang Z, Huang C (1994) Identification and characterization of kink motifs in 1-palmitoyl-2-oleoyl-phosphatidylcholines: a molecular mechanics study. Biophys J 66:2005-2018

Lide DR (2001-2002) CRC handbook of chemistry and physics. Chapman and Hall, Boca Raton

Litman BJ, Lewis EN, Levin IW (1991) Packing characteristics of highly unsaturated bilayer lipids: Raman spectroscopic studies of multilamellar phosphatidylcholine dispersions. Biochemistry 30:313-319

Macdonald PM, Sykes BD, McElhaney RN, Gunstone FD (1985) Fluorine-19 nuclear magnetic resonance studies of lipid fatty acyl chain order and dynamics in Acholeplasma laidlawii B membranes. Orientational order in the presence of a series of positional isomers of cis-octadecenoic acid. Biochemistry 24:177-184

Marsh D (1990) CRC handbook of lipid bilayers. CRC Press, Boca Raton

McElhaney RN (1986) The use of differential scanning calorimetry and differential thermal analysis in studies of model and biological membranes. Biochim Biophys Acta 864:361-421

Metso AJ, Mattila JP, Kinnunen PKJ (2004) Characterization of the main transition of dinervonoylphosphocholine liposomes by fluorescence spectroscopy. Biochim Biophys Acta 1663:222-231

Mou J, Yang J, Huang C, Shao Z (1994) Alcohol induces interdigitated domains in unilamellar phosphatidylcholine bilayers. Biochemistry 33:9981-9985

Müller DJ, Engel A (1997) The height of biomolecules measured with the atomic force microscope depends on electrostatic interactions. Biophys J 73:1633-1644 
Özdirekcan S, Nyholm TKM, Raja M, Rijkers DTS, Liskamp RMJ (2008) Influence of trifluoroethanol on membrane interfacial anchoring interactions of transmembrane $\alpha$-helical peptides. Biophys J 94:1315-1325

Petrache HI, Tristram-Nagle S, Harries D, Kucerka N, Nagle JF, Parsegian VA (2006) Swelling of phospholipids by monovalent salt. J Lipid Res 47:302-309

Rauls M, Bartosch K, Kind M, Kuch S, Lacmann R, Mersmann A (2000) The influence of impurities on crystallization kinetics-a case study on ammonium sulfate. J Cryst Growth 213:116-128

Rinia HA, Kik RA, Demel RA, Snel MME, Killian JA, Van der Eerden JPJM, De Kruijff B (2000) Visualization of highly ordered striated domains induced by transmembrane peptides in supported phosphatidylcholine bilayers. Biochemistry 39:5852-5858

Rinia HA, Boots JWP, Rijkers DTS, Kik RA, Snel MME, Demel RA, Killian JA, Van der Eerden JPJM, De Kruijff B (2002) Domain formation in phosphatidylcholine bilayers containing transmembrane peptides: specific effects of flanking residues. Biochemistry 41:2814-2824

Scarpelli F, Drescher M, Rutters-Meijneke T, Holt A, Rijkers DTS, Killian JA, Huber M (2009) Aggregation of transmembrane peptides studied by spin-label EPR. J Phys Chem B 113:12257-12264

Shao Z, Mou J, Czajkowsky DM, Yang J, Yuan J-Y (1996) Biological atomic force microscopy: what is achieved and what is needed. Adv Phys 45:1-86

Siegel DP, Cherezov V, Greathouse DV, Koeppe RE II, Killian JA, Caffrey M (2006) Transmembrane peptides stabilize inverted cubic phases in a biphasic length-dependent manner: implications for induced membrane fusion. Biophys J 90:200-211
Sparr E, Ganchev DN, Snel MME, Ridder ANJA, Kroon-Batenburg LMJ, Chupin V, Rijkers DTS, Killian JA, De Kruijff B (2005) Molecular organization in striated domains induced by transmembrane $\alpha$-helical peptides in dipalmitoyl phosphatidylcholine bilayers. Biochemistry 44:2-10

Van den Brink-Van der Laan E, Killian JA, De Kruijff B (2004) Nonbilayer lipids affect peripheral and integral membrane proteins via changes in the lateral pressure profile. Biochim Biophys Acta 1666:275-288

Vitovič P, Kresák S, Naumann R, Schiller SM, Lewis RNAH, McElhaney RN, Hianik T (2004) The study of the interaction of a model $\alpha$-helical peptide with lipid bilayers and monolayers. Bioelectrochemistry 63:169-176

Vollhardt D (2007) Effect of unsaturation in fatty acids on the main characteristics of Langmuir monolayers. J Phys Chem C 11:6805-6812

Williams WP, Cunningham BA, Wolfe DH, Derbyshire GE, Mant GR, Bras W (1996) A combined SAXS/WAXS investigation of the phase behaviour of di-polyenoic membrane lipids. Biochim Biophys Acta 1284:86-96

Yarrow F, Kuipers BWM (2011) AFM study of the thermotropic behavior of supported DPPC bilayers with and without the model peptide WALP23. Chem Phys Lipids 164:9-15

Yeagle PL (2005) The structure of biological membranes. CRC Press, Boca Raton

Yun H, Choi YW, Kim NJ, Sohn D (2003) Physicochemical properties of phosphatidylcholine (PC) monolayers with different alkyl chains, at the air/water interface. Bull Korean Chem Soc 24:377-383 